\title{
Estimativa da área foliar do girassol por método não destrutivo
}

\author{
Leonardo Angelo de Aquino $\left({ }^{1 *}\right)$; Valdeir Celestino dos Santos Júnior ( $\left.{ }^{2}\right)$; João Victor Santos Guerra \\ $\left({ }^{2}\right)$; Marileide Moreira Costa $\left({ }^{2}\right)$
}

(') Universidade Federal de Viçosa (UFV), Campus Rio Paranaíba, Rodovia BR 354, Km 310, 38810-000 Rio Paranaíba (MG), Brasil. (2) Instituto Federal do Norte de Minas Gerais, Campus Januária, Fazenda São Geraldo, s/n, Bom Jardim, 39480-000 Januária (MG), Brasil.

(*) Autor correspondente: leonardo.aquino@ufv.br

Recebido: 13/out./2010; Aceito: 14/mar./2011

\section{Resumo}

Métodos de fácil execução, rápidos e não destrutivos, que possibilitem estimar a área foliar com precisão, são importantes para avaliar o crescimento das plantas nas condições de campo. Objetivou-se no presente trabalho, ajustar equações para estimar a área do limbo foliar e a área das folhas do girassol, em função das medidas lineares do limbo e do número de folhas por planta, incluindo a verificação da possibilidade de modelos comuns para as cultivares BR-122 e M-734. Seis plantas de cada cultivar nos estádios de início de florescimento e de florescimento pleno foram coletadas. As áreas dos limbos foliares foram determinadas por método direto. Foram medidos o comprimento ao longo da nervura principal e a largura de forma perpendicular à inserção do limbo no pecíolo. Foram ajustados os modelos linear, quadrático, cúbico, exponencial e potencial. Os modelos potenciais $\hat{Y}_{i}=1,6329 X_{i}^{1,7164}$ e $\hat{Y}_{i}=0,5405 X_{i}^{1,0212}$ com a utilização, respectivamente, das medidas da largura e do produto largura e comprimento são os mais adequados para estimar a área do limbo foliar do girassol. O modelo $\hat{Y}_{i}=5,1014 X_{i}^{2,4383}$ permite estimar com precisão a área foliar total do girassol em função do número de folhas por planta. $A$ precisão das equações ajustadas para as estimativas da área do limbo foliar ou de folhas por planta não é reduzida quando se ajustam modelos comuns às cultivares BR-122 e M-734.

Palavras-chave: Helianthus annuus L., crescimento de planta, alometria.

\section{Estimates of sunflower leaf area by a non-destructive method}

\begin{abstract}
Simple, rapid, and non-destructive methods that help to estimate leaf area accurately are very important to assess plant growth under field conditions. The objective of this study was to adjust equations to estimate the area of a single sunflower leaf and also the area of total leaves per plant as a function of linear measurements of them. It was also verified the possibility of using common models for the sunflower cultivars BR-122 and M-734. Six plants of each cultivar in the early stage of flowering and full flowering were harvested. The area of each leaf was determined by the direct method. The length along the midrib and the width perpendicular to leaf insertion in the petiole were determined. Linear, quadratic, cubic, exponential, and potential models were adjusted. The potential models $\hat{Y}_{i}=1.6329 X_{i}^{1,7164}$ and $\hat{Y}_{i}=0.5405 X_{i}^{1,0212}$ using the width measurements and width $x$ length, respectively, were the most adequate to estimate the leaf area. The model $\hat{Y}_{i}=5.1014 X_{i}^{2,4383}$ allowed to precisely estimate the total leaf area according to the number of leaves per plant. The accuracy of the adjusted equations to estimate each leaf are and total leaves area was not reduced when common models were adjusted to the two cultivars used.
\end{abstract}

Key words: Helianthus annuus L., plant growth, allometry.

O girassol (Helianthus annuus L.) é uma planta caracterizada por sua versatilidade industrial e adaptabilidade climática. Considerada uma das principais oleaginosas do mundo, responde por aproximadamente $13 \%$ da produção mundial de óleo (Amabile et al., 2002).

Um dos processos mais importantes que determinam a produtividade de uma cultura é a fotossíntese, que depende da interceptação da energia luminosa. Esta depende, dentre outros fatores, do índice de área foliar (IAF), que expressa a relação entre a área das folhas e aquela ocupada pela planta (Rouphael et al., 2007; Maldaner et al., 2009).

A quantificação da área foliar pode ser realizada por métodos diretos ou indiretos. O método direto é destrutivo. Consiste em coletar as folhas e passá-las em um aparelho integrador de área foliar. Este método tem o inconveniente de necessitar de maior área das parcelas experimentais, haja vista que plantas serão coletadas durante o experimento. Além disso, os aparelhos integradores de área foliar têm custo elevado 
e o processo de determinação da área foliar é moroso (Zucoloto et al., 2008).

Métodos de fácil execução, rápidos e não destrutivos que estimem a área foliar com precisão são importantes para avaliar o crescimento das plantas nas condiçôes de campo (Zucoloto et al., 2008). Estes métodos trazem vantagens como o fato de que as amostragens poderão ser feitas com as mesmas plantas durante o ciclo de desenvolvimento, reduzindo o erro experimental (Posse et al., 2009).

Estudos têm mostrado a viabilidade de estimar a área foliar e a biomassa foliar seca a partir de medidas lineares do limbo (comprimento, largura ou seu produto) em cultivos agrícolas (RouphaEL et al., 2007; Ramos et al., 2008) e em plantas daninhas (BiAnco et al., 2007).

Equaçôes para estimar a área foliar do girassol a partir de medidas lineares do limbo foram desenvolvidas por Rouphael et al. (2007) e Maldaner et al. (2009). No entanto, o formato das folhas das diferentes cultivares, os estádios fenológicos e os fatores ambientais podem interferir na precisão da estimativa da área foliar a partir das medidas lineares do limbo (Ramos et al., 2008).

Além da área de uma folha, muitas vezes é interessante a quantificação da área foliar por planta e da biomassa seca das folhas e da parte aérea. Em bananeira foi possível o ajuste de equaçóes para estimar a área foliar total e/ou biomassa seca da parte aérea a partir do diâmetro de caule (Zucoloto et al., 2008).

Objetivou-se no presente trabalho ajustar equaçóes para estimar a área do limbo foliar e das folhas, em função das medidas lineares do limbo e do número de folhas por planta, respectivamente, e verificar a possibilidade de uso de modelos comuns para as cultivares de girassol BR-122 e M-734.

O experimento foi realizado no município de Januária (MG), em condiçôes de campo, localizado na latitude de $15^{\circ} 28^{\prime} 55^{\prime \prime} \mathrm{S}$ e longitude de $44^{\circ} 22^{\prime} 41^{\prime \prime} \mathrm{W}$, altitude de $474 \mathrm{~m}$.

A semeadura foi realizada em 20/4/2010. Adotou-se o espaçamento entre plantas de $70 \times 33 \mathrm{~cm}$, utilizando as cultivares Dow Agroscience M-734 e EMBRAPA BR122, em 10 linhas de $30 \mathrm{~m}$ para cada cultivar. Foram consideradas úteis as seis linhas centrais, menos $2 \mathrm{~m}$ de cada extremidade.

Realizou-se a fertilização de acordo com os resultados da análise física e química do solo e recomendaçôes para a cultura (Ribeiro et al., 1999). Utilizou-se irrigação por aspersão convencional. Realizaram-se duas capinas para controle de plantas daninhas, não sendo necessária a aplicação de métodos químicos para controle de pragas e doenças na cultura.

Nos estádios fenológicos R1 e R6, foram retiradas seis plantas ao acaso na área útil de cada cultivar. O estádio R1 é caracterizado por $50 \%$ das plantas no início da emissão do botão floral e o R6 por 50\% das plantas com capítulos e todas as flores tubulares desenvolvidas (CASTIGLIONI et al., 1997). Os estádios R1 e R6 ocorreram, respectivamente, aos 37 e 57 DAE (dias após a emergência) na cultivar BR 122 e aos 46 e 69 DAE na M-734.

As folhas foram destacadas do caule e contadas separadamente por planta coletada. Cada folha foi separada em limbo e em pecíolo. A área dos limbos foliares foi determinada por método direto. Foram tomadas as medidas de comprimento (C) e da largura (L) do limbo foliar. O comprimento foi medido ao longo da nervura principal e a largura, de forma perpendicular, à inserção do pecíolo no limbo.

Procederam-se às análises de regressão entre as variáveis dependentes (área do limbo ou área foliar total por planta) $\mathrm{e}$ as independentes (largura, comprimento, seu produto $\mathrm{L}^{*} \mathrm{C}$ e número de folhas por planta). Ajustaram-se os modelos linear, quadrático, cúbico, potencial e exponencial. Utilizou-se para ajuste das equaçôes de regressão o "software” Excel, Versão $2007^{\circledR}$ (Microsoft Corporation, 2007). Um resumo das medidas lineares, de área dos limbos e da área e do número de folhas por planta utilizadas para geração dos modelos está inserido na tabela 1 .

As análises foram realizadas para os dados das cultivares separadamente e, posteriormente, para o conjunto de dados das duas cultivares. Foram escolhidos os modelos lineares com coeficiente de determinação $\left(\mathrm{R}^{2}\right)$ maior que $80 \%$. Os demais modelos foram escolhidos quando superaram o $\mathrm{R}^{2}$ do modelo linear e a menor raiz do quadrado médio do erro - RQME (JansSEn E Heuberger, 1995):

$$
\operatorname{RQME}=\left[\sum\left(s_{\mathrm{i}}-\mathrm{o}_{\mathrm{i}}\right)^{2} / \mathrm{N}\right]^{2}
$$

em que "s" é o valor estimado, "o" o valor observado e N o número de observaçóes para geração do modelo $(\mathrm{N}=220$, 229 e 449 folhas para a cultivar BR-122, M-734 e para o conjunto de folhas de ambas as cultivares respectivamente). Determinou-se o $\mathrm{R}^{2}$ na regressão linear simples entre os valores observados e os estimados pelos modelos escolhidos com base nos critérios mencionados.

Dois modelos com maior coeficiente de determinaçáo de geração do modelo para estimativa da área foliar (AF) e menor RQME para o conjunto de dados das duas cultivares foram comparados aos modelos $\mathrm{AF}=6,72+0,65 \mathrm{~L}^{2}$ $\left(\mathrm{R}^{2}=0,978\right)$, proposto por RouphaEL et al. (2007) e AF = $1,7582 \mathrm{~L}^{1,7067}\left(\mathrm{R}^{2}=0,983\right)$, proposto por MALDANER et al. (2009). Na comparação dos modelos, utilizou-se um conjunto de 449 folhas das cultivares estudadas.

Foram obtidos modelos para estimativa da área do limbo foliar das cultivares BR-122 e M-734 e para o conjunto de dados de ambas as cultivares a partir das medidas de comprimento, da largura e do produto dessas medidas (Tabela 2). Nos modelos obtidos para a cultivar BR-122 ocorreu menor RQME comparados aos obtidos para a M-734. Diferenças das cultivares com relação ao formato, ao número de folhas e à área foliar total por planta 
justificam as diferenças quanto à precisão dos modelos na estimava da área foliar (Tabela 1).

$\mathrm{O}$ ajuste de modelo para estimativa da área do limbo foliar com os dados conjuntos das cultivares foi adequado, possibilitando a obtenção de modelos com $\mathrm{R}^{2}>0,90$, considerado como valor adequado (MALDANER et al., 2009). Os modelos gerados para estimativa da área do limbo foliar a partir da largura foram mais precisos que aqueles com base no comprimento para ambas as cultivares (Tabela 2). Resultados semelhantes foram obtidos em meloeiro (Lopes et al., 2007) e em girassol (RouphaEL et al., 2007; MALDANER et al., 2009).

A regressão entre a área foliar e o produto $\mathrm{C}^{*} \mathrm{~L}$ possibilitou o ajuste de modelos com maior $\mathrm{R}^{2}$ e menor RQME para as duas cultivares e na análise com os dados agrupados. Quanto maior o valor de $\mathrm{R}^{2}$ e menor o de RQME, maior é a precisão do modelo (JansSen e Heuberger, 1995).
Apesar do aumento da precisão na estimativa da área foliar a partir do produto $\mathrm{C}^{*} \mathrm{~L}$, há aumento do tempo gasto em campo para medição dessas variáveis. Modelos que usem apenas a medida de $\mathrm{C}$ ou L são mais adequados por reduzir à metade o número de mediçóes (MaLDANER et al., 2009).

Os dois modelos para estimativa da área do limbo foliar com maior $\mathrm{R}^{2}$ e menor RQME para o conjunto de dados das cultivares foram os potenciais com variável independente $\mathrm{L}$ ou $\mathrm{C}^{*} \mathrm{~L}$ (Tabela 2). O modelo gerado com a variável independente $\mathrm{L}$ proporcionou RQME similar aos modelos propostos por RouphaEL et al. (2007) e àquele proposto por MaLDANER et al. (2009), ambos modelos potenciais (Figura 1). No modelo com a variável independente $\mathrm{C}^{*} \mathrm{~L}$ notou-se menor RQME comparado aos modelos dos autores anteriormente citados.

Os modelos propostos para estimativa da área do limbo foliar por Rouphael et al. (2007) e por Maldaner

Tabela 1. Valores médios e desvio-padrão da área foliar total (AFT) e do número de folhas por planta (NF), da largura (L), do comprimento (C) e da área individual de folha (AF) para cada época de amostragem e cultivar de girassol

$\begin{array}{lcccccc}\text { Cultivar } & \text { Estádio } & \begin{array}{c}\text { AFT } \\ \left(\mathbf{c m}^{2} \text { planta-1) }^{-1}\right.\end{array} & \begin{array}{c}\text { NF } \\ \text { (unid.) }\end{array} & \begin{array}{c}\text { L } \\ (\mathbf{c m})\end{array} & \begin{array}{c}\text { C } \\ (\mathbf{c m})\end{array} & \begin{array}{c}\text { AF } \\ \left(\mathbf{c m}^{2}\right)\end{array} \\ \text { BR-122 } & \mathrm{R}^{*} & 4800 \pm 1600 & 16,3 \pm 1,9 & 18,5 \pm 5,5 & 21,8 \pm 4,8 & 264 \pm 126 \\ & \mathrm{R}^{* *} & 6278 \pm 2229 & 19,2 \pm 3,4 & 22,7 \pm 6,4 & 24,0 \pm 5,2 & 339 \pm 161 \\ \text { M-734 } & \mathrm{R} 1 & 8159 \pm 2572 & 19,8 \pm 2,0 & 24,8 \pm 7,0 & 25,9 \pm 5,5 & 418 \pm 204 \\ & \text { R6 } & 10419 \pm 3137 & 22,3 \pm 2,5 & 24,8 \pm 6,0 & 26,6 \pm 5,0 & 465 \pm 205\end{array}$

*R1 - 50\% das plantas com botăo floral; ** R6 - 50\% das plantas com capítulos e todas as flores tubulares desenvolvidas (CASTIGLIONI et al., 1997).

Tabela 2. Modelos para estimativa da área do limbo foliar do girassol $\left(\mathrm{cm}^{2}\right)$ em função das medidas máximas neste órgão, da largura (L $\mathrm{cm})$, do comprimento $(\mathrm{C}-\mathrm{cm})$ e de seu produto $\left(\mathrm{C}^{*} \mathrm{~L}\right)$

\begin{tabular}{|c|c|c|c|c|}
\hline Cultivar & Variável Independente (X) & Modelo Estatístico & RQME* & $\mathbf{R}^{\mathbf{2}^{* *}}$ \\
\hline \multirow{7}{*}{ BR 122} & \multirow{2}{*}{$\mathrm{C}$} & $\hat{Y}_{i}=27,06 X_{i}-317,03\left(R^{2}=0,86\right)-$ Linear & 55 & 0,86 \\
\hline & & $\hat{Y}_{i}=0,2499 X_{i}^{2,2418}\left(R^{2}=0,91\right)-$ Potencial & 50 & 0,89 \\
\hline & \multirow{3}{*}{ L } & $\hat{Y}_{i}=23,36 X_{i}-159,10\left(R^{2}=0,90\right)-$ Linear & 51 & 0,90 \\
\hline & & $\hat{Y}_{i}=2,2199 X_{i}^{2,6053}\left(R^{2}=0,95\right)$ - Potencial & 43 & 0,92 \\
\hline & & $\hat{Y}_{i}=0,4116 X_{i}^{2}+5,3687 X_{i}\left(R^{2}=0,92\right)-$ Quadrático & 43 & 0,92 \\
\hline & \multirow{2}{*}{$C^{*} \mathrm{~L}$} & $\hat{Y}_{i}=0,5852 X_{i}+8,0115\left(R^{2}=0,93\right)-$ Linear & 39 & 0,93 \\
\hline & & $\hat{Y}_{i}=0,7591 X_{i}^{0,9621}\left(R^{2}=0,96\right)-$ Potencial & 39 & 0,93 \\
\hline \multirow{7}{*}{ M-734 } & \multirow{3}{*}{ C } & $\hat{Y}_{i}=35,94 X_{i}-501,90\left(R^{2}=0,86\right)-$ Linear & 78 & 0,86 \\
\hline & & $\hat{Y}_{i}=0,1429 X_{i}^{2,4345}\left(R^{2}=0,92\right)-$ Potencial & 69 & 0,89 \\
\hline & & $\hat{Y}_{i}=25,59 e^{0,1033 x_{i}}\left(R^{2}=0,91\right)-$ Exponencial & 73 & 0,88 \\
\hline & \multirow{2}{*}{$\mathrm{L}$} & $\hat{Y}_{i}=29,56 X_{i}-292,09\left(R^{2}=0,88\right)-$ Linear & 72 & 0,88 \\
\hline & & $\hat{Y}_{i}=1,2709 X_{i}^{1,8025}\left(R^{2}=0,94\right)-$ Potencial & 68 & 0,89 \\
\hline & \multirow{2}{*}{$C^{*} \mathrm{~L}$} & $\hat{Y}_{i}=0,7029 X_{i}-39,00\left(R^{2}=0,92\right)-$ Linear & 57 & 0,92 \\
\hline & & $\hat{Y}_{i}=0,4111 X_{i}^{1,0667}\left(R^{2}=0,96\right)-$ Potencial & 57 & 0,92 \\
\hline \multirow{9}{*}{$\begin{array}{l}\text { BR } 122 \\
\text { e } \\
\text { M-734 }\end{array}$} & \multirow{3}{*}{ C } & $\hat{Y}_{i}=32,65 X_{i}-429,84\left(R^{2}=0,86\right)-$ Linear & 73 & 0,86 \\
\hline & & $\hat{Y}_{i}=0,1748 X_{i}^{2,3651}\left(R^{2}=0,92\right)-$ Potencial & 63 & 0,90 \\
\hline & & $\hat{Y}_{i}=24,18 e^{0,1049 x i}\left(R^{2}=0,92\right)-$ Exponencial & 64 & 0,89 \\
\hline & \multirow{3}{*}{ L } & $\hat{Y}_{i}=26,81 X_{i}-237,07\left(R^{2}=0,88\right)-$ Linear & 67 & 0,88 \\
\hline & & $\hat{Y}_{i}=1,6329 X_{i}^{1,7164}\left(R^{2}=0,95\right)-$ Potencial & 61 & 0,90 \\
\hline & & $\hat{Y}_{i}=0,5477 X_{i}^{2}+2,8239 X_{i}\left(R^{2}=0,90\right)-$ Quadrático & 60 & 0,90 \\
\hline & \multirow{3}{*}{$C^{*} \mathrm{~L}$} & $\hat{Y}_{i}=0,6653 X_{i}-22,55\left(R^{2}=0,93\right)-$ Linear & 53 & 0,93 \\
\hline & & $\hat{Y}_{i}=0,5405 X_{i}^{1,0212}\left(R^{2}=0,96\right)-$ Potencial & 53 & 0,93 \\
\hline & & $\hat{Y}_{i}=9,3 \cdot 10^{-5} X_{i}^{2}+0,5582 X_{i}\left(R^{2}=0,93\right)-$ Quadrático & 51 & 0,93 \\
\hline
\end{tabular}

${ }^{*}$ Raiz quadrada do quadrado médio do erro; ${ }^{* *}$ Coeficiente de determinação da Regressão Linear de Primeiro Grau entre os valores reais e estimados da variável resposta. 
et al. (2009) ofereceram estimativas precisas da área foliar (Figura 1). No modelo proposto por RoupHAEL et al. (2007), foi usado um conjunto de dados de genótipos cultivados na Europa, e no modelo proposto por Maldaner et al. (2009), utilizaram-se os dados das cultivares Aguará e Helio 358. Os dados obtidos neste trabalho se referem às cultivares BR-122 e M-734. Esse fato sugere que os modelos podem ser utilizados para estimativa da área do limbo foliar em genótipos diferentes daqueles usados neste trabalho, com boa precisão nas estimativas.

Foram obtidos modelos com bom ajuste para estimativa da área foliar usando o número de folhas por planta para as cultivares individualmente e para os
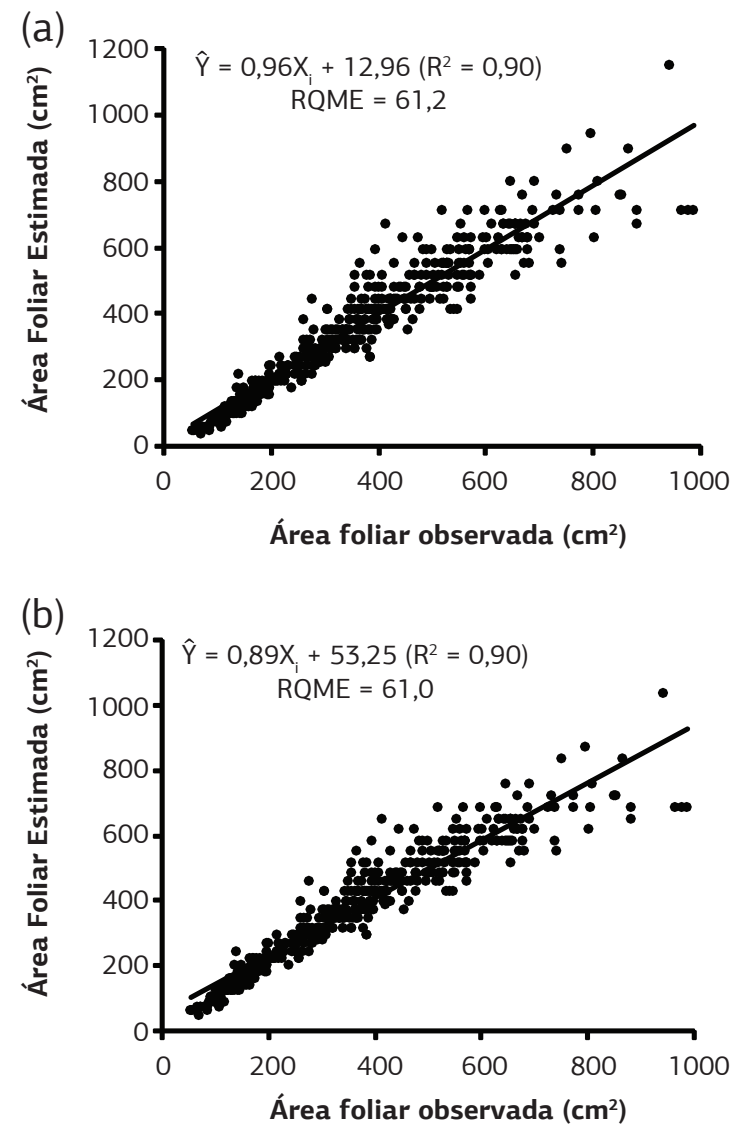

dados analisados em conjunto (Tabela 3). Em bananeira prata anã, o modelo para estimativa da área foliar por planta inclui além do número de folhas por planta, o comprimento e a largura da terceira folha (Zucoloto et al., 2008). Os modelos obtidos para o girassol são mais simples e dependem apenas da contagem do número de folhas.

Conclui-se que é possível estimar a área do limbo foliar por meio de medidas lineares deste e a área foliar por planta a partir do número de folhas. A precisão das equaçóes ajustadas para as estimativas da área do limbo foliar ou de folhas por planta não é reduzida quando se utilizam modelos comuns às cultivares BR-122 e M-734.

(c)

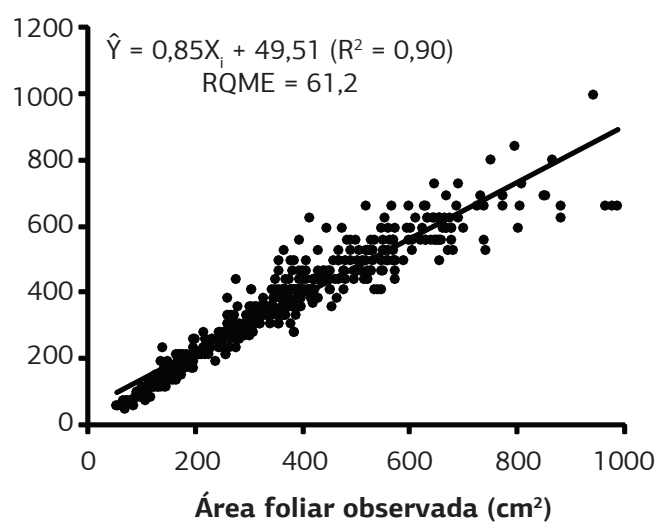

(d)

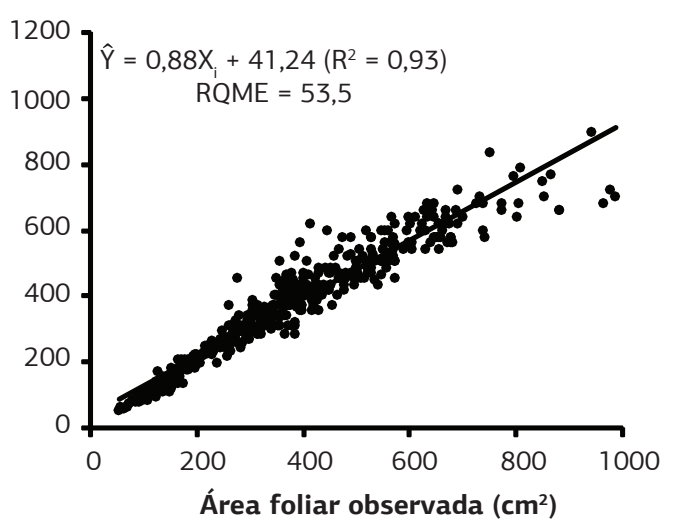

Figura 1. Comparação dos modelos para estimativa da área do limbo foliar em girassol: (a) Rouphael et al. (2007); (b) Maldaner et al. (2009); (c) modelo proposto com base na largura do limbo foliar; (d) modelo proposto com base no produto da largura e comprimento do limbo foliar.

Tabela 3. Modelos para estimativa da área foliar por planta no girassol $\left(\mathrm{cm}^{2}\right.$ planta $\left.{ }^{-1}\right)$ em função do número de folhas por planta

\begin{tabular}{|c|c|c|c|}
\hline Cultivar & Modelo Estatístico & RQME* $^{*}$ & $\mathbf{R}^{\mathbf{2}^{* *}}$ \\
\hline \multirow{2}{*}{ BR 122} & $\hat{Y}_{i}=641,79 X_{i}-5852,36\left(R^{2}=0,89\right)-$ Linear & 663 & 0,89 \\
\hline & $\hat{Y}_{i}=9,27 X_{i}^{2}+284,98 X_{i}-2516,67\left(R^{2}=0,89\right)-$ Quadrático & 655 & 0,89 \\
\hline \multirow{2}{*}{ M-734 } & $\hat{Y}_{i}=1145,48 X_{i}-14804,07\left(R^{2}=0,93\right)-$ Linear & 772 & 0,93 \\
\hline & $\hat{Y}_{i}=17,56 X_{i}^{2}+371,61 X_{i}-3694,64\left(R^{2}=0,93\right)-$ Quadrático & 757 & 0,93 \\
\hline \multirow{3}{*}{$\begin{array}{l}\text { BR } 122 \\
\text { e } \\
M-734\end{array}$} & $\hat{Y}_{i}=928,64 X_{i}-10593,53\left(R^{2}=0,88\right)-$ Linear & 1058 & 0,88 \\
\hline & $\hat{Y}_{i}=26,06 X_{i}^{2}-113,68 X_{i}-433,40\left(R^{2}=0,89\right)-$ Quadrático & 1002 & 0,89 \\
\hline & $\hat{Y}_{i}^{\prime}=5,1014 X_{i}^{2,4383}\left(R^{2}=0,91\right)-$ Potencial & 1008 & 0,89 \\
\hline
\end{tabular}

* Raiz quadrada do quadrado médio do erro; ${ }^{* *}$ Coeficiente de determinação da Regressão Linear de Primeiro Grau entre os valores reais e estimados da variável resposta. 


\section{AGRADECIMENTOS}

Ao $\mathrm{CNPq}$, pelo auxílio financeiro, e à Fapemig, pelo auxílio financeiro e pela concessão de bolsa de Iniciação Científica.

\section{REFERÊNCIAS}

AMABILE, R.F.; FERNANDES, F.D.; SANZONOWICZ, C. Girassol como alternativa para o sistema de produção no Cerrado. EMBRAPA: CNPSO, 2002. 3p. (Circular Técnica, 20)

BIANCO, S.; BIANCO, M.S.; PAVANI, M.C.M.D.; DUARTE, D.J. Estimativa da área foliar de Ipomoea hederifolia em Ipomoea nil Roth. usando dimensōes lineares do limbo foliar. Planta Daninha, v.25, p.325-329, 2007.

CASTIGLIONI, V.B.R.; BALLA, A.; CASTRO, C.; SILVEIRA, J.M. Fases de desenvolvimento da planta de girassol. EMBRAPA: CNPSO, 1997. 24p. (Documentos n. 58)

JANSSEN, P.H.M.; HEUBERGER, P.S.C. Calibration of process: oriented models. Ecological Modelling, v.83, p.55-56, 1995.

LOPES, S.J.; BRUM, B.; SANTOS, V.J.; FAGA, E.B.; LUZ, G.L.; MEDEIROS, S.L.P. Estimativa da área foliar de meloeiro em estádios fenológicos por fotos digitais. Ciência Rural, v.37, p.1153-1156, 2007.
MALDANER, I.C.; HELDWEIN, A.B.; LOOSE, L.H.; LUCAS, D.D.P.; GUSE, F.I.; BERTOLUZZI, M.P. Modelos de determinação não-destrutiva da área foliar em girassol. Ciência Rural, v.39, p.1356-1361, 2009.

POSSE, R.P.; SOUSA, E.F.; BERNARDO, S.; PEREIRA, M.G.; GOTTARDO, R.R. Total leaf area of papaya trees estimated by a nondestructive method. Scientia Agricola, v.66, p.462-466, 2009.

RAMOS, A.; BOVI, M.L.A.; FOLEGATTI, M.V.; DIOTTO, A.V. Estimativas da área foliar e da biomassa aérea da pupunheira por meio de relações alométricas. Horticultura Brasileira, v.26, p.138-143, 2008.

RIBEIRO, A.C.; GUIMARÁES, P.T.G.; ALVAREZ, V.H. Recomendação para o uso de corretivos e fertilizantes em Minas Gerais: 5a Aproximação. Viçosa: Comissáo de Fertilidade do Solo do Estado de Minas Gerais, 1999. 359p.

ROUPHAEL, Y.; COLLA, G.; FANASCA, S.; KARAM, F. Leaf area estimation of sunflower leaves from simple linear measurements. Photosynthetica, v.45, p.306-308, 2007.

ZUCOLOTO, M.; LIMA, J.S.S.; COELHO, R.I. Modelo matemático para estimativa da área foliar total de bananeira 'Prata-Anã'. Revista Brasileira de Fruticultura, v.30, p.11521154, 2008 